\title{
Escape acquisition following inescapable shock in the rat
}

\author{
JOHN E. KELSEY \\ Indiana University, Bloomington, Indiana 4740 I
}

\begin{abstract}
Male rats which had received approximately 21 min of pulsed, inescapable tail shock during a 6-h session in a wheel-turn chamber were markedly deficient in acquisition of an FR 2 crossing escape response in a shuttlebox when first tested 22 or $70 \mathrm{~h}$ later (Experiments 1 and 2). Rats which had received identical amounts and patterns of escapable/avoidable shock, however, were not deficient (Experiment 1). Preventing wheel-turn responses during the inescapable shocks prevented the occurrence of the subsequent escape deficit, whereas reducing the feedback provided for the first crossing response of the FR 2 requirement enhanced the deficit (Experiment 3). These data can be best explained by the learned helplessness hypothesis and indicate that the types of responses available and made during the inescapable shocks are more important than previously indicated.
\end{abstract}

Exposure to inescapable shocks in several species can severely interfere with subsecuent acquisition of escape responses• (e.g., Maier \& Seligman, 1976). Maier and Seligman and their co-workers argue that many of these interference effects occur because the organisms learn during the inescapable shocks that onset and offset of shock are independent of their own behavior (e.g., Maier \& Seligman, 1976). As a result, the organisms initiate fewer active escape responses in a new situation and find it more difficult to learn a response-shock contingency even when successful responses are emitted.

Although many of the interference effects produced by inescapable shocks can be explained by this learned helplessness hypothesis (Maier \& Seligman, 1976), considerable controversy still surrounds the validity of this explanation (Levis, 1976). For example, inescapable shocks can produce interference effects with characteristics which are inconsistent with the learned helplessness hypothesis (Weiss, Glazer, \& Pohorecky, 1975). Furthermore, it has been argued that the interference effects can be more adequately and parsimoniously explained in terms of the development of competing responses such as freezing or immobility (Anisman \& Waller, 1973; Bracewell \& Black, 1974; Glazer \& Weiss, $1976 \mathrm{~b})$ or in terms of decreased motor activation due to depletion of brain norepinephrine (Weiss et al., 1975).

The present experiments examine some aspects of this controversy by determining whether an inter-

This research was supported, in part, by PHS RR 7031-10 Biomedical Sciences Support Grant to Indiana University. I would like to thank Lee Hoyman and Eric Haseltine for their assistance with the statistical analyses. Requests for reprints should be sent to John E. Kelsey, Department of Psychology, Indiana University, Bloomington, Indiana 47401. ference effect that has characteristics predicted from the learned helplessness hypothesis can be produced in rats if procedures designed to minimize both the development and transfer of competing responses are used. Most investigators who have obtained interference effects in rats (e.g., Bracewell \& Black, 1974; Glazer \& Weiss, 1976a, b; Maier, Albin, \& Testa, 1973) have used nonpulsed grid shock, whose current density animals can frequently reduce by adopting freezing responses which are incompatible with active escape (McClelland \& Colman, 1967). Furthermore, by applying shocks to the same part of the rat's body, frequently in the same apparatus, in both phases of these experiments, many investigators have encouraged successful transfer of any competing responses which did develop during exposure to inescapable shocks (e.g., Anisman \& Waller, 1973; Seligman \& Beagley, 1975). To reduce some of these problems, I initially presented approximately 21 min of pulsed shock to the immobilized tails of rats during a 6-h session in a chamber containing a wheel which could be turned. At least $22 \mathrm{~h}$ later, the ability of these rats to escape pulsed footshock in a shuttlebox by making two successive crossing responses (FR 2) was examined.

To be considered an example of learned helplessness, any interference effect produced by these procedures should have the following characteristics. Since helplessness is assumed to be the effect of experience with uncontrollable events, the interference effect should be caused by exposure to inescapable shocks and not by exposure to identical durations and patterns of escapable/avoidable shock (Experiment 1). Although the interference effect in dogs is clearly due to prior exposure to uncontrollable shocks (Maier, 1970; Seligman \& Maier, 1967), it is not as clear that this is a characteristic of the interference effects observed in rats. Two addi- 
tional predictions flow from the aspect of the hypothesis stating that the escape deficit depends on having learned during the inescapable shocks that responses and shocks are uncorrelated. First, since the effects of learning are usually long lasting, the interference effect should still be apparent when first examined several days after exposure to inescapable shocks (Experiments 2 and 3). Although interference effects lasting at least 7 days have been obtained in rats (Glazer \& Weiss, 1976a; Seligman \& Beagley, 1975), other investigators have found the effects to be transient (Anisman, 1975; Weiss et al., 1975). Second, because of response generalization, the interference effect should be larger if the organisms learn during the inescapable shocks that responses that are qualitatively similar to the subsequently required escape response are uncorrelated with shocks than if they only learn that markedly dissimilar responses are uncorrelated (Experiment 3). Since the type of responses available and made during the inescapable shocks have not been frequently examined (Glazer \& Weiss, 1976a) or manipulated (Bracewell \& Black, 1974), a test of this latter prediction should be useful, not only in evaluating the learned helplessness hypothesis, but in establishing boundary conditions for this hypothesis. Finally, since experience with response-independent shock is presumed to make the learning of new response-shock contingencies more difficult, increasing the difficulty of the escape contingency to be learned should enhance the magnitude of the interference effect (Experiment 3). Although this prediction has been elegantly confirmed by Maier and Testa (1975), replication using different procedures would be desirable.

\section{EXPERIMENT 1}

The major requirement of the learned helplessness hypothesis is that an interference effect be attributable to prior experience with shocks that were uncontrollable. Therefore, a yoked design was used to determine if exposure to inescapable shocks, using the procedures outlined above, would interfere with acquisition of a difficult (FR 2) escape response in the shuttlebox $22 \mathrm{~h}$ later, and if, as predicted, exposure to identical durations and patterns of escapable/avoidable shocks would not.

\section{Method}

Subjects. The subjects were 31 naive male Sprague-Dawley rats weighing from 290 to $460 \mathrm{~g}$ at the beginning of experimentation. The rats had ad-lib access to food and water except during testing and were initially housed two to a cage in a colony that was illuminated from 7 a.m. to 7 p.m. each day.

Apparatus. The three identical, clear Plexiglas wheel-turn chambers were similar to those described by Weiss (1971). The grid floor was made of 1-cm-diam aluminum rods spaced $2.5 \mathrm{~cm}$ apart (center to center). The wheel, which could only be turned downward, had two notches spaced $180^{\circ}$ apart. Whenever these notches passed a miniature switch attached to the chamber, a response was recorded. Immediately above each wheel was a $55 \cdot \mathrm{V}$ (No. 1835) light bulb which was powered by $28 \mathrm{~V}$ so that it was only dimly illuminated. Each chamber was housed inside its own ventilated, sound-attenuating shell and was illuminated by a dim houselight. White noise of $60 \mathrm{~dB}$ SPL was supplied through speakers at the top of each chamber to mask extraneous sounds.

The two $20 \times 49 \times 31 \mathrm{~cm}$ shuttleboxes were made of galvanized steel except for the front and tops, which were made of clear Plexiglas. Each box was divided into two $20 \times 24.3 \mathrm{~cm}$ interconnecting chambers by a black Masonite partition which had a $7 \times 8 \mathrm{~cm}$ opening in the center flush with the grid floor. The floor was made of .6-cm-diam stainless steel rods separated by $2 \mathrm{~cm}$ (center to center). This floor pivoted in the center and was supported on each end by adjustable weights and miniature switches. A 28-V Sonalert (Mallory) unit was located $8 \mathrm{~cm}$ above the center partition of each box and generated a relatively pure tone of $2,900 \mathrm{~Hz}$ at $70 \mathrm{~dB}$ SPL. Twenty-eight-volt (No. 1819) light bulbs were positioned $3 \mathrm{~cm}$ above the ceiling of both chambers so that each chamber could be illuminated separately. Each shuttlebox was housed inside its own ventilated soundattenuating shell, which was illuminated by a dim houselight. A speaker located at the top of each shell supplied a masking white noise of $65 \mathrm{~dB}$ SPL.

The three constant ac current shock sources consisted of $5,000-\mathrm{V}$ ac transformers in series with limiting resistors. The output of one shocker was connected to two shock electrodes in the wheel-turn chambers. The output of another shocker was connected to the grids of one shuttlebox, and the output of the third shocker was connected to the grids of the remaining shuttlebox. The grids of each shuttlebox were wired in parallel with NE-2 bulbs, and the galvanized steel walls were connected to the center grids. The current in the wheel-turn chambers was measured when 10-KQ resistors (pseudorats) were placed in series with the electrodes. The current in the shuttleboxes was measured across six consecutive grids with no rat on the grid floor. The current in all shockers was pulsed at the rate of $1.5 / \mathrm{sec}$ with an on time of $.4 \mathrm{sec}$.

Procedure. The rats were randomly divided into three groups before being placed in the wheel-turn chambers. Rats in the escape/avoidance group were able to escape and avoid shock by turning the wheel. Each rat in the yoked group was placed in a second chamber and received the same number, duration, intensity, and pattern of shocks as received by an escape/avoidance rat. Rats in the control group were placed in the third wheel-turn chamber but were not shocked.

Each rat was placed in the appropriate chamber, and its tail, which had been cleaned with alcohol, was pulled out through the hole in the back panel. In order to prevent the rat from pulling its tail into the apparatus, a plastic disk was slipped over the tail and secured with tape. Two 3-cm-long 18-ga stainless steel hypodermic needles served as shock electrodes and were taped about $2.5 \mathrm{~cm}$ apart and on opposite sides of the tail. To prevent the rat from rolling and shorting or disconnecting the electrodes, its tail was then taped to a plastic rod attached to an immobile base. Electrode paste was applied and replenished when necessary throughout the 6-h session. The electrodes of the escape/avoidance and yoked rats were wired in series to a single shock source. The electrodes of the control rats were disconnected.

Shocks were scheduled to occur on a free-operant avoidance schedule. Once shock was presented, the escape/avoidance rat was required to escape the shock by turning the wheel in a downward direction until it tripped the miniature switch (less than or equal to one-half a revolution of the wheel). Each escape response terminated the shock for both the escape/avoidance and yoked rats, illuminated the bulb located above the wheel in all three chambers for $1 \mathrm{sec}$, and reset the timer controlling the response-shock interval. Each wheel-turn response made by the escape/avoidance rat during the response-shock interval 
also illuminated the three bulbs and reset the interval, thus postponing the next shock. Similar wheel-turn responses by the yoked and control rats were recorded, but had no effect on shock onset or offset and did not illuminate the bulbs.

During the first $30 \mathrm{~min}$, the response-shock interval was $15 \mathrm{sec}$ and the shock intensity was $.8 \mathrm{~mA}$. No attempt was made to shape wheel-turn responses. After $30 \mathrm{~min}$, the response-shock interval was increased to $30 \mathrm{sec}$ for the remainder of the session and the shock intensity was increased to $1.0 \mathrm{~mA}$. Shock intensity was increased to $1.2 \mathrm{~mA}$ at the end of $2 \mathrm{~h}$ and to $1.4 \mathrm{~mA}$ after $4 \mathrm{~h}$. At the end of the 6-h session, the rats were removed from the chambers, weighed, and housed one to a cage.

Approximately $22 \mathrm{~h}$ later, these rats were allowed to escape shock in the shuttleboxes. The weights on each end of the grid floor were adjusted for each rat so that a crossing response was automatically recorded when the rat had moved the middle of its body approximately $13 \mathrm{~cm}$ (6.5 grids) into the opposite chamber. Each of the 30 trials was initiated by simultaneous activation of the Sonalert and illumination of the light bulb in the half of the box occupied by the rat. Ten seconds later, .7-mA current was applied to the grid floor and the steel walls. During the first five trials, each rat was required to cross into the opposite chamber in order to terminate the shock, light, and tone. Trials on which avoidance responses (crossing during the 10-sec CS-US interval), occurred were not counted.

On the remaining 25 trials, the rats were required to cross to the opposite chamber and then back to the original side (FR 2 requirement) in order to escape shock and terminate the light and tone. Although avoidance responses were still possible, none of the rats in any of the experiments made even one crossing response during the CS-US interval during these FR 2 trials. To make this FR 2 crossing response easier to perform, the rats were provided with exteroceptive feedback following completion of the first crossing response. First, the light stimulus was turned on in the compartment into which the rat had just moved and was turned off in the original compartment. Because the current did not initially extend past the seventh grid in the opposite chamber and did not switch completely into that chamber until $.3 \mathrm{sec}$ after completion of the first crossing, a brief shock-free period also usually followed completion of the first crossing. If a rat had not completed the FR 2 escape requirement within $35 \mathrm{sec}$, the shock, tone, and light were automatically terminated. The rats were allowed to cross freely between the two chambers during the fixed $45-\mathrm{sec}$ intertrial interval, but these responses had no programmed effect. All testing was carried out during the day.

Statisties. Most of the data were analyzed by a single-factor analysis of variance. The escape latencies in the shuttleboxes were analyzed in blocks of five trials by multivariate analysis of variance. Further comparisons following a significant main effect were made using the Newman-Keuls procedure. All $p$ values are for two-tailed tests.

\section{Results}

During the 6-h session in the wheel-turn chambers, the 10 escape/avoidance rats acquired the wheel-turn response rapidly and received an average of 326.8 shocks (of a maximum possible of 720), which they escaped in an average of $3.8 \mathrm{sec}$. As expected, these rats made more wheel-turn responses $(\overline{\mathrm{X}}=3,790.7)$ than the 10 yoked rats $(\bar{X}=1,138.1 ; p<.01)$, which, in turn, made more responses than the 11 unshocked control rats $(\overline{\mathrm{X}}=25.6 ; \mathrm{p}<.05)$. Both the escape/avoidance and yoked rats lost more weight ( $\overline{\mathrm{X}} \mathrm{s}=25.4$ and $23.5 \mathrm{~g}$, respectively) than the control rats $(\overline{\mathrm{X}}=12.8 \mathrm{~g} ; \mathrm{p}<.01)$.

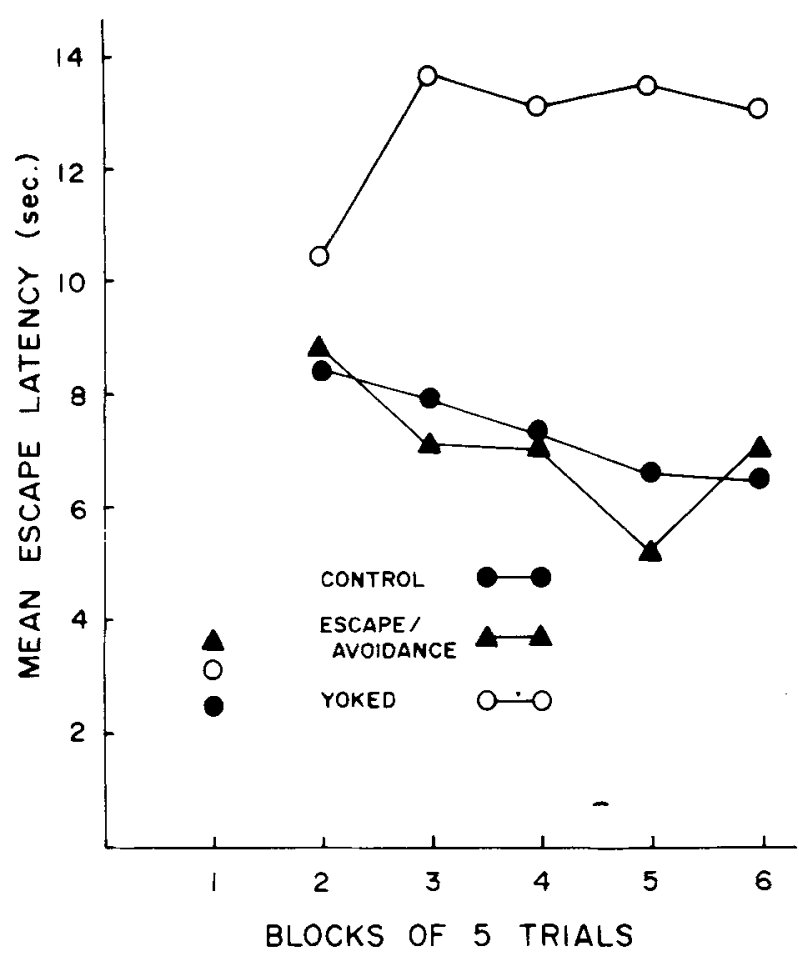

Figure 1. Acquisition of FR 2 escape in the shuttlebox 1 day after exposure to shocks in the wheel-turn chambers. Only one (FR 1) response was required on the first block of five trials. The remaining five blocks show the latency to complete the entire FR 2 requirement.

The rats which were allowed to escape and avoid shock in the wheel-turn boxes subsequently learned to escape shock in the shuttleboxes as rapidly and reliably as the previously unshocked control rats (Figure 1). The yoked rats, which had received inescapable shocks, escaped shock during the first five FR 1 trials as rapidly as the other two groups (Figure 1). However, during the subsequent 25 FR 2 trials, the yoked rats escaped more slowly $(p<.04$ and $\mathrm{p}<.03$ ) and successfully escaped on fewer trials $\overline{(X}=20.6)$ than both the control $\bar{X}=23.0 ; p<.02)$ and escape/avoidance rats $(\bar{X}=24.4 ; \mathrm{p}<.05)$, respectively (Figure 1 ). Two yoked rats performed as well as the control and escape/avoidance rats, whereas four yoked rats (and one control) required an average of $20.8 \mathrm{sec} /$ trial to escape shock (FR 2 trials only) and escaped on an average of only 13.0 trials. The remaining four yoked rats required an average of $10.9 \mathrm{sec} /$ trial to escape and escaped on an average of 24.0 trials.

The magnitude of the interference effect did not depend on the duration of inescapable shock received. The five yoked rats which had received the least amount of shock in the wheel-turn chambers $\bar{X}=423.6 \mathrm{sec})$ subsequently escaped as slowly in the shuttleboxes $(X=13.0 \mathrm{sec} /$ trial; $F R 2$ trials only) as the five yoked rats $(\bar{X}=14.0 \mathrm{sec} /$ trial $)$ which received the most shock $(\bar{X}=2,060.1 \mathrm{sec})$. 


\section{Discussion}

This experiment demonstrated that exposure to an average of $21 \mathrm{~min}$ of pulsed, inescapable tailshock during a 6-h session in a wheel-turn chamber caused deficits in subsequent acquisition of an FR 2 crossing escape response in a shuttlebox. Most important, exposure to an identical duration and pattern of escapable/avoidable shock had no effect on subsequent escape in the shuttlebox. These results represent only the second demonstration of an interference effect in rats that can be completely attributed to prior experience with shocks that were uncontrollable (Glazer \& Weiss, 1976b). Thus, an interference effect having the major characteristic required by the learned helplessness hypothesis can be produced using these procedures.

In addition to supporting the learned helplessness hypothesis, these results do not support an alternative learned inactivity hypothesis (Glazer \& Weiss, 1976b). Glazer and Weiss (1976a) found that an interference effect would occur only when rats were exposed to inescapable shocks lasting at least $5 \mathrm{sec}$. Based on casual observations that the rats frequently stopped struggling after 3 or $4 \mathrm{sec}$ of shock, they argued that shock termination which occurred after this period would, thus, adventitiously reinforce the rats for not struggling. Consequently, they argued that their rats were deficient in subsequent acquisition of an active escape response, not because the rats were helpless and less capable of learning, but because they had previously learned an incompatible response of remaining inactive in the presence of shock.

My results, on the other hand, indicate that the magnitude of the escape deficit did not depend on the total duration of inescapable shock received or on the average duration received per shock. The five yoked rats which received the least amount of inescapable shock received an average of only $2.9 \mathrm{sec} / \mathrm{shock}$, but were just as deficient in subsequent escape acquisition as the remaining five yoked rats which received an average of $4.1 \mathrm{sec} / \mathrm{shock}$. Although it is undoubtedly true that even the former five yoked rats received some shocks that were more than $5 \mathrm{sec}$ in duration, these must have been few in number and probably occurred early in the session. Thus, if these rats were similar to those of Glazer and Weiss (1976a), they should have been actively struggling when most of the shocks terminated and, thus, should have learned to become even more active rather than inactive. In fact, since these rats were receiving pulsed tailshock, which decreases the tendency to become inactive, it is likely that even rats which received shocks longer than $5 \mathrm{sec}$ in duration were frequently still struggling when shock terminated. These results thus suggest that an interference effect can be produced even when the in- escapable shocks are not sufficiently long for the rats to become inactive prior to shock termination. Thus, the interference effect obtained in this experiment is not likely to be due to learned inactivity as postulated by Glazer and Weiss (1976b).

\section{EXPERIMENT 2}

If the interference effect obtained in Experiment 1 depends on learning, as required by the learned helplessness hypothesis, then the interference effect should also be observable when escape acquisition is first examined several days following exposure to the inescapable shocks. Therefore, the main purpose of this experiment was to determine if exposure to inescapable shocks would interfere with escape acquisition both 22 and $70 \mathrm{~h}$ later.

Since Experiment 1 demonstrated that the interference effect was caused only by prior exposure to inescapable shocks, the escape/avoidance group was no longer essential and the inescapable shocks could be subsequently presented according to a standardized schedule. However, this schedule must be designed so that its effects can also be unambiguously attributed to the uncontrollability of the shock. Most investigators have seemingly ignored this essential qualification. Seligman and his co-workers (Seligman \& Beagley, 1975; Seligman, Rossellini, \& Kozak, 1975), for example, after finding that exposure to escapable shock interfered with subsequent escape acquisition less than yoked exposure to inescapable shock, subsequently used a standardized sequence that produced two to three times as much inescapable shock as previously generated by the escape rats. The assumption that the effects of these increased durations and different patterns of inescapable shocks were due to the fact that they were uncontrollable may not be warranted. A more rational procedure would consist of constructing the standardized sequence of shocks on the basis of the pattern of shocks actually produced by the escape rats. Therefore, rats in this and the subsequent experiment were exposed to inescapable shocks programmed to reproduce the median number, duration, and pattern of shocks generated by the escape/ avoidance rats of Experiment 1.

\section{Method}

Subjects. The rats were 39 naive male Sprague-Dawley rats that had been housed individually in our colony for 4 to 6 weeks and weighed from 300 to $490 \mathrm{~g}$ at the beginning of experimentation.

Apparatus. The three wheel-turn chambers, two shuttleboxes, and three shock sources described in Experiment 1 were used.

Procedure. The number and duration of shocks received by the escape/avoidance rats during the 6-h wheel-turn sessions of Experiment 1 were divided into four periods consisting of the first $30 \mathrm{~min}$ of the session, the next $11 / 2 \mathrm{~h}$, the next $2 \mathrm{~h}$, and the last $2 \mathrm{~h}$. Four separate film tapes. were then punched, each 
reproducing the median frequency, duration, and approximate variability of shocks produced by the escape-avoidance rats during one of the four periods. In addition, the first 30-min tape was punched so that a substantial reduction in shocks, approximating that produced by most escape/avoidance rats, occurred as the 30 min elapsed.

The procedure during the wheel-turn sessions was essentially identical to that of Experiment 1, except that the occurrence and duration of shocks were controlled by the four film tapes. Thus, in each triplet of rats, there were usually an unshocked control rat and two rats wired in series and receiving the same inescapable shocks. In contrast to the preceding experiment, the lights above the wheels were never illuminated.

Approximately 22 or $70 \mathrm{~h}$ after the termination of these 6-h sessions, the rats were tested for acquisition of escape in the shuttleboxes as described in Experiment 1, with the exception that two crossing responses (FR 2) were required to escape shock on all 30 trials. The addition of a printout counter enabled us to record separately the escape latencies for both the first and second crossing responses on each trial.

Statistics. With one exception, all comparisons between groups were made by means of a t test adjusted for unequal variance (Hays \& Winkler, 1971). Response latencies in the shuttleboxes were analyzed in blocks of five trials by multivariate analysis of variance. All $\mathrm{p}$ values are for two-tailed tests.

\section{Results}

As in Experiment 1, the 14 inescapably shocked rats subsequently escaped shock in the shuttleboxes more slowly ( $\mathrm{p}<.02$; Figure 2 ) and successfully escaped on fewer trials $(\bar{X}=23.4)$ than the 10 control rats $(\bar{X}=29.9 ; p<.03)$ when tested $22 \mathrm{~h}$ after the 6-h session in the wheel-turn chambers. Most of this effect was attributable to slower escape latencies on the first crossing response of the FR 2

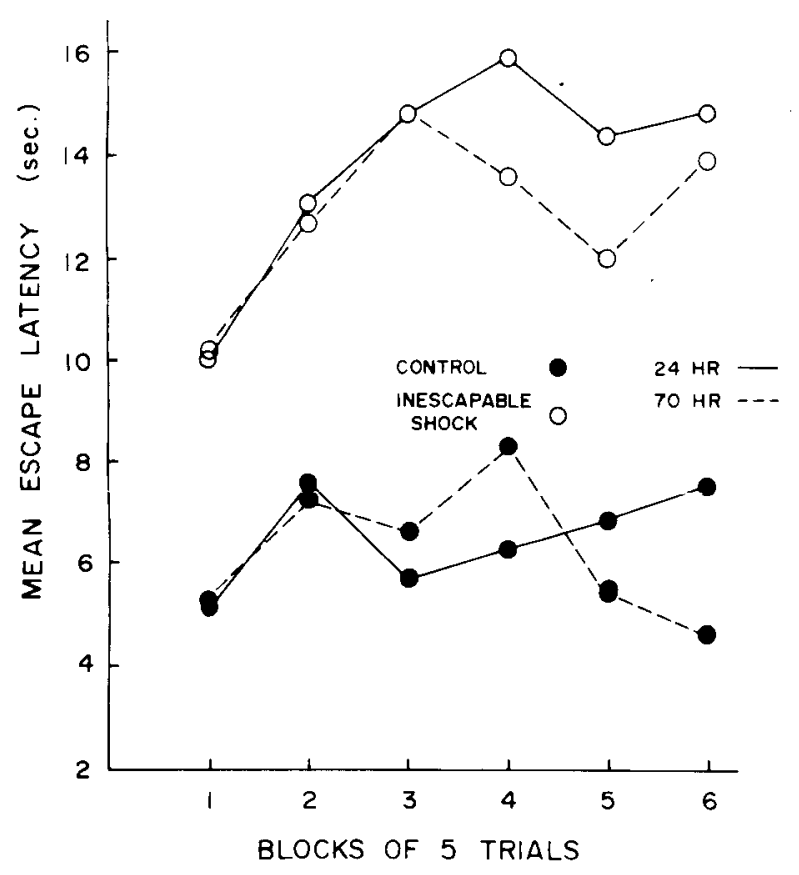

Figure 2. Acquisition of FR 2 escape in the shuttlebox 1 day $(24 \mathrm{~h})$ and 3 days $(70 \mathrm{~h})$ after exposure to inescapable shocks in the wheel-turn chambers. requirement $(p<.02)$, as the groups did not differ significantly in their latency to make the second crossing on trials when the first crossing had occurred $(\mathrm{p}<.10$; Figure 2$)$.

When tested 3 days $(70 \mathrm{~h})$ after the session in the wheel-turn chambers, the nine inescapably shocked rats also escaped shock in the shuttleboxes more slowly than the six previously unshocked control rats $(p<.03$; Figure 2$)$. Although the previously shocked rats also tended to escape on fewer trials $(\bar{X}=26.8)$ than the controls $(\bar{X}=29.8)$, this effect was not statistically significant. There were no significant differences between the two control groups or between the two inescapably shocked groups.

During the session in the wheel-turn chambers, the 23 inescapably shocked rats received an average of 348.5 shocks totaling $1,278.4 \mathrm{sec}$. These rats made more wheel-turn responses $(\overline{\mathrm{X}}=1,960.9)$ and lost more weight $(\overline{\mathrm{X}}=27.2 \mathrm{~g})$ than the 16 unshocked control rats $(\overline{\mathrm{X}}=71.9$ responses; $\mathrm{p}<.001$; and $\overline{\mathrm{X}}=17.8 \mathrm{~g}, \mathrm{p}<.001)$. Although the inescapably shocked rats regained more of this lost weight $(\bar{X}=14.6 \mathrm{~g})$ during the 3 -day recovery period than the comparable rats after only 1 day of recovery ( $\bar{X}=4.6 \mathrm{~g} ; \mathrm{p}<.01$ ), they had still not regained as much as the controls $(\bar{X}=18.8 \mathrm{~g} ; \mathrm{p}<.01)$ by the time of the shuttlebox tests.

\section{Discussion}

This experiment demonstrated that exposure to a standard sequence of inescapable shocks significantly interfered with subsequent FR 2 escape when the rats were first tested either 22 or $70 \mathrm{~h}$ after the inescapable shocks. In fact, the magnitude of the interference effect observed at $22 \mathrm{~h}$ was essentially identical to that caused by yoked exposure to the inescapable shocks from which this standardized sequence was generated (Experiment 1). Since Experiment 1 demonstrated that exposure to these same numbers, durations, and patterns of escapable shock did not interfere with subsequent escape, it can be unambiguously concluded that the effects of the shocks used in this experiment were due to the fact that they were uncontrollable. This conclusion, which has not been warranted in previous investigations, justifies continued use of this procedure.

The finding that the inescapably shocked rats still escaped more slowly than the control rats when first examined 3 days after the inescapable shocks indicates that the interference effect produced by this procedure, like the similar long-lasting interference effects produced by Glazer and Weiss (1976a) and Seligman et al. (1975), depends on learning as required by the learned helplessness hypothesis. Furthermore, these findings clearly distinguish these effects from interference effects which are quite transient (e.g., Anisman, 1975; Weiss et al., 1975) 
and, thus, cannot be attributed to learned helplessness.

\section{EXPERIMENT 3}

This experiment was designed to examine two additional implications of the learned helplessness hypothesis. First, if the interference effect reflects difficulty in learning about new response-shock contingencies, as postulated by this hypothesis, then making the escape contingency more difficult to learn should enhance the interference effect. To reexamine this prediction, which has been elegantly confirmed by Maier and Testa (1975), the effects of prior inescapable shocks were examined on acquisition of an escape response which was made more difficult to learn by reducing the exteroceptive feedback provided the rat after making the first crossing of the FR 2 escape requirement.

Second, since the learned helplessness hypothesis states that the interference effect is due to having learned during the inescapable shocks that responses and shocks were uncorrelated, the magnitude of the interference effect should depend on how effectively this learning occurs. Although the implications of this aspect of the theory have never been explicitly stated, the magnitude of the escape deficit should depend, therefore, on the number and types of responses that are made during the inescapable shocks. For example, animals should be more likely to learn that their responses and shocks are independent if they make many responses during the inescapable shocks, and thus have many opportunities to learn, than if they make few responses. Similarly, because of response generalization, the magnitude of the escape deficit should be greater if the responses which the animals learn are uncorrelated with shocks are qualitatively similar to the subsequently required escape response than if they are markedly dissimilar. Both the FR 2 crossing escape response and the wheel-turn response appear to be similar emitted or voluntary responses, which are quite different from the shock-elicited, species-specific struggling responses which are frequently made by rats in the wheel-turn chambers. Therefore, it would be expected that preventing the occurrence of the emitted response of wheel-turning during the inescapable shocks would decrease the probability that rats would learn that voluntary responses are uncorrelated with shock, and, consequently, would decrease the magnitude of the subsequent FR 2 escape deficit. This prediction was tested by examining the effects of locking the wheels during the inescapable shocks on subsequent escape acquisition.

\section{Method}

Subjects. The subjects were 28 naive male Sprague-Dawley rats housed individually in our colony for 3 to 5 weeks and weighing 360 to $460 \mathrm{~g}$ at the beginning of experimentation.

Apparatus. The three wheel-turn chambers and two shuttleboxes described in Experiment 1 were used.

Procedure. The rats were exposed to inescapable shock (or no shock) in the wheel-turn chambers, as in Experiment 2. However, for nine inescapably shocked rats and six control rats, the wheels were locked so that they could not be turned during the 6-h sessions.

All rats were tested for escape acquisition in the shuttleboxes $70 \mathrm{~h}$ later using the procedures described in Experiment 2, with the exception of the following modifications designed to reduce the exteroceptive feedback provided after completion of the first crossing response of the FR 2 requirement. The overhead lights, which signaled both trial onset and the completion of the first crossing, were not used. Since Maier and Testa (1975) had indicated that a brief offset of shock following the first crossing response of the FR 2 requirement reduced the interference effect, two changes were made to decrease the probability that this brief termination would occur. First, a crossing response was recorded when the rat moved the middle of its body only $9 \mathrm{~cm}$ ( 4.5 grids), as opposed to the previous $13 \mathrm{~cm}$ (6.5 grids), into the opposite chamber. Second, the shock was extended an additional $4 \mathrm{~cm}$ ( 2 grids) into the opposite chamber, well past the point at which the rat would record a response. Because these manipulations made the escape response more difficult to acquire, only a single crossing response was required to escape shock on the first five trials. Two (FR 2) crossing responses were required on each of the remaining 25 trials as in the previous experiments.

\section{Results}

Because locking the wheels did not significantly affect the subsequent escape behavior of the previously unshocked control rats (Figure 3), the data of the two control groups were combined for pur-

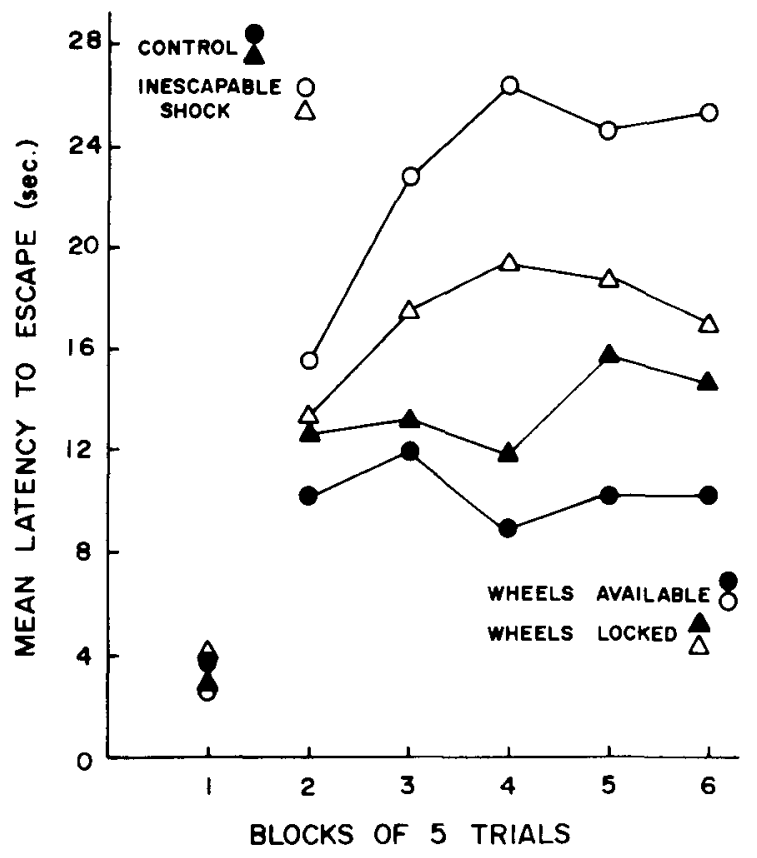

Figure 3. Effects of manipulations of the escape contingency on acquisition of FR 2 escape in the shuttlebox 3 days after exposure to inescapable shocks given while the wheel-turn response was available or while the wheels were locked. Only one (FR 1) response was required on the first block of five trials. The remaining five blocks show the latency to complete the entire FR 2 requirement. 
pose of statistical analysis. Reducing the exteroceptive feedback for the first crossing response made the FR 2 escape response in the shuttlebox more difficult to acquire as the 11 control rats escaped more slowly, but not less reliably, than the controls in the previous experiments ( $p<.05$; Figure 3 ).

Although the inescapably shocked rats subsequently escaped as rapidly as controls on the first five FR 1 trials, the eight rats previously exposed to inescapable shocks when the wheel-turn response was available were severely deficient in their ability to escape shock on the subsequent 25 FR 2 trials (Figure 3). During the FR 2 trials, these rats escaped more slowly $(\mathrm{p}<.03$ and $\mathrm{p}<.05)$ and successfully escaped on fewer trials $(\overline{\mathrm{X}}=13.6)$ than the control rats of this experiment $\bar{X}=23.4 ; p<.01)$ and the inescapably shocked rats of Experiment 2 (adjusted for the last 25 FR 2 trials; $p<.05$ ), respectively. Although the data are not shown, these inescapably shocked rats were slower than the controls to make the first crossing rèsponse on each FR 2 trial $(p<.03)$. They were not, however, significantly slower to make the second crossing response, even when trials on which the first crossing did not occur were excluded. This severe deficit appeared to be essentially all or none, with two of the inescapably shocked rats escaping as rapidly and reliably as controls and the remaining six requiring an average of $29.8 \mathrm{sec} /$ trial to escape shock (FR 2 trials only) and successfully escaping on an average of only 10 FR 2 trials.

Although the interference effect observed in the rats exposed to inescapable shocks when the wheels were unlocked was significantly more severe than in previous experiments, the interference effects observed in rats exposed to inescapable shocks when the wheels were locked was not enhanced. In fact, these latter rats did not escape significantly more slowly or less reliably than the control rats, and they successfully escaped on more FR 2 trials $(\bar{X}=20.2)$, although not more rapidly, than the inescapably shocked rats which were allowed to turn the wheels (Figure 3; $\mathrm{p}<.05$ ).

As in previous experiments, the inescapably shocked rats made more wheel-turn responses $(\bar{X}=3,005.6)$ than the control rats $(\bar{X}=146.8$; $\mathrm{p}<.001)$. Both groups of inescapably shocked rats lost more weight in the wheel-turn chambers $(\bar{X}=27.6 \mathrm{~g})$ than the unshocked control rats $\overline{(\bar{X}}=$ $17.7 \mathrm{~g} ; \mathrm{p}<.01)$. In spite of the 3 -day recovery period, only the control rats had regained this lost weight by the day of escape testing.

\section{Discussion}

This experiment demonstrated that reducing the exteroceptive feedback provided for completing the first crossing response of the FR 2 escape requirement in the shuttlebox markedly enhanced the interference effect caused by prior exposure to inescapable shocks. While the control rats escaped as reliably (although more slowly) as the controls in previous experiments, the rats exposed to inescapable shocks when a wheel-turn response was available subsequently failed to escape shock on two to three times as many trials as the inescapably shocked rats in the previous experiments.

This reduction of feedback was presumed to make the escape contingency more difficult to learn. However, since this reduction also caused the rats of both groups to expose themselves to more shock, it is possible that it was this factor, and not the altered contingencies per se, which enhanced the interference effect. This possibility seems unlikely, however, because Maier and Testa (1975) found that increasing the amount of shock received in the shuttlebox in the absence of altered contingencies did not enhance the interference effect. Therefore, it is reasonable to suggest that the reduction of feedback enhanced the interference effect, because it made the escape contingency more difficult to learn. Since the learned helplessness hypothesis states that the interference effect reflects a deficit in learning new contingencies, it predicts that making the escape contingency more difficult to learn should enhance this effect. Thus, the finding that reducing the feedback enhanced the escape deficit supports the learned helplessness hypothesis.

Although the interference effect was enhanced when the rats could turn the wheels during the inescapable shocks, the escape deficit observed when the rats were prevented from turning the wheels during these shocks was not significant. Because the rats explicitly trained to turn the wheels in Experiment 1 subsequently escaped shock as rapidly as previously unshocked controls, it is unlikely that locking the wheels eliminated the interference effect by retarding acquisition of a competing response associated with wheel-turning. Furthermore, this effect of locking the wheels cannot be easily explained by alternative hypotheses which suggest that inescapable shocks cause a deficit in subsequent acquisition of an active escape response by enhancing the development of incompatible freezing or immobility responses (Anisman \& Waller, 1973; Bracewell \& Black, 1974; Glazer \& Weiss, 1976a, b). Since the active wheel-turn response itself is incompatible with freezing, preventing the occurrence of this highly probable active response should have enhanced the development of immobility responses and, thus, enhanced the subsequent interference effect. Thus, the finding that locking the wheels eliminated the interference effect indicates that these alternative hypotheses cannot adequately account for the interference effects observed in the present experiments.

On the other hand, this observation is quite consistent with the learned helplessness hypothesis that 
the escape deficit depends on having learned during the inescapable shocks that responses and shocks are uncorrelated. In fact, these data appear to help define the boundary conditions of this hypothesis by demonstrating that learning that certain types of responses are uncorrelated with shock is more likely to interfere with subsequent acquisition of a particular escape response than is learning that other types of responses are uncorrelated. In the introduction to this experiment, I suggested that the emitted or voluntary wheel-turn response appears to be qualitatively more similar to the FR 2 crossing escape response than are the shock-elicited, speciesspecific struggling responses which also occur reliably in the wheel-turn chambers. Thus, the finding that preventing the rats from learning that wheelturning and shocks are uncorrelated eliminated the subsequent interference effect supports my contention that the magnitude of the escape deficit should be greater if the organism learns that responses that are qualitatively similar to the subsequently required escape response are uncorrelated with shock than if it only learns about dissimilar responses.

Irrespective of the validity of this interpretation, these data indicate that the types of responses available and made during exposure to inescapable shocks are more important than stated or implied by the learned helplessness hypothesis. Increased attention to these responses should prove beneficial, not only because of the relevance of these data to competing response theories, but because these data should help establish the boundary conditions of the learned helplessness hypothesis.

\section{GENERAL DISCUSSION}

Exposure of rats to approximately $21 \mathrm{~min}$ of pulsed, inescapable tailshock during a 6-h period in a wheel-turn chamber markedly interfered with subsequent acquisition of an FR 2 crossing response to escape pulsed grid shock in a shuttlebox. This interference effect had the most important characteristics required by the learned helplessness hypothesis. The observation that exposure to identical amounts and patterns of escapable/avoidable shock did not interfere with subsequent escape acquisition demonstrated that this interference effect was completely dependent on prior exposure to shocks which the rats could not control (Experiment 1). The finding that the rats were deficient in escape acquisition when first tested both 1 and 3 days following the inescapable shocks indicated that this interference effect was not transient and probably depended on learning (Experiments 2 and 3). Furthermore, decreasing the exteroceptive feedback for the first crossing of the FR 2 escape response enhanced the magnitude of this interference effect (Experiment 3). Since this reduc- tion of feedback appeared to make the escape contingency more difficult to learn, this finding is consistent with a hypothesis which suggests that the interference effect reflects a difficulty in learning new response-shock contingencies. Although these three results essentially replicate previous observations (e.g., Glazer \& Weiss, 1976a, b; Maier \& Seligman, 1976), they further support and extend the generality of the learned helplessness hypothesis by demonstrating that the major predictions of this hypothesis can be confirmed using different procedures.

Moreover, this study produced a new result which, in addition to supporting the learned helplessness hypothesis, appears to help establish boundary conditions for this hypothesis. Preventing the rats from making the highly probable, voluntary wheel-turn responses during the inescapable shocks prevented the occurrence of the interference effect (Experiment 3 ). This effect could not be attributed to the retardation of acquisition of competing responses. It was argued that this result suggests that, because of response generalization, the magnitude of the escape deficit will be greater when the responses that the animals learn are uncorrelated with shocks during the inescapable shocks are qualitatively similar to the subsequently required escape response than when they are markedly dissimilar. Although quite consistent with the learned helplessness hypothesis, this important, new finding indicates that the kinds of responses available and made during the inescapable shocks are more important than explicitly stated by this hypothesis.

In addition to directly supporting the learned helplessness hypothesis, one of the major strengths of these data is that they cannot be easily explained by the major alternative hypotheses. Most of these alternatives suggest that interference with escape is not due to an inability to learn, but is due to the development and transfer of responses, such as freezing or immobility, which are incompatible with active escape (e.g., Anisman \& Waller, 1973). Three hypotheses suggest that rats may learn to become immobile during the inescapable shocks. First, the rats might be able to reduce the current density of nonpulsed dc grid shock by remaining immobile (McClelland \& Colman, 1967). Second, Bracewell and Black (1974) argued that highly restrained rats may learn to become immobile because struggling against the restraint may enhance the pain of the shocks. Third, Glazer and Weiss (1976a) argued that if the duration of the inescapable shocks is sufficiently long (greater than $5 \mathrm{sec}$ ) the rats would usually stop struggling, and this inactivity would, thus, be adventitiously reinforced by subsequent shock termination. In addition, Weiss et al. (1975) argued that exposure to intense (4 mA) inescapable 
shocks may transiently increase the difficulty of performing effortful responses by temporarily depleting the brain of the putative neurotransmitter, norepinephrine.

It is most unlikely, however, that inactivity could have developed in the present study by any of these proposed mechanisms. First, as argued in the introduction, it is unlikely that any available response, especially freezing, would have been effective in reducing the current density of the pulsed, inescapable shock to the immobilized tail. Second, because the rats of the present study were restrained only by their tails and could easily move without pain, Bracewell and Black's (1974) argument that highly restrained rats may learn to become immobile because struggling causes pain is not applicable. Third, my observation that rats consistently exposed to inescapable shocks which were less than $5 \mathrm{sec}$ in duration ( $\mathrm{X}=2.9 \mathrm{sec}$ ) were deficient in subsequent escape acquisition does not support the conclusion of Glazer and Weiss (1976a) that, in order to produce an interference effect, rats must be exposed to inescapable shocks of durations that are sufficiently long to elicit inactivity. Finally, the motor activation deficit hypothesis of Weiss et al. (1975) cannot account for the observation that the interference effect could still be observed when first examined 3 days after the mild inescapable shocks. Any depleted brain levels of norepinephrine almost certainly would have recovered during that time period (Bliss, Ailion, \& Zwanziger, 1968; Stone, 1973).

Moreover, if these alternative hypotheses were correct, locking the wheels during the inescapable shocks should have enhanced the interference effect, because preventing the occurrence of this highly probable, active response should have enhanced the development of inactivity. Instead, locking the wheels eliminated the interference effect. Furthermore, even if an incompatible response developed and became conditioned to the stimuli of the wheelturn boxes, it would probably have transferred only weakly to the shuttleboxes, which have considerably different stimulus (and response) characteristics. In fact, explicit training of the incompatible response of standing and turning a wheel produced absolutely no interference with subsequent escape in the shuttleboxes (Experiment 1). Finally, during the initial FR 2 trials in the shuttleboxes, the previously inescapably shocked rats were not freezing, but were escaping as rapidly as the controls. Thus, it appears mos! unlikely that the interference effects observed in the present study can be attributed to the development and transfer of incompatible freezing or immobility responses.

Since the interference effect observed in these experiments has several characteristics which can be predicted from the learned helplessness hypothesis and which cannot at present be adequately explained by the major alternative hypotheses, these findings provide substantial support for the validity of the learned helplessness hypothesis. However, these data also suggest that this hypothesis presently is incomplete. The finding that locking the wheels during the inescapable shocks prevented the interference effect from occurring indicates that the kinds of responses available and made during the inescapable shocks are more important than stated by the learned helplessness hypothesis. Second, the shape of the escape acquisition curves appears to depend on the particular escape responses examined. Seligman and Maier (1967), for example, found that the escape deficit in inescapably shocked dogs was maximal on the first few trials, and Maier et al. (1973) suggested that inescapable shocks would interfere only with escape responses that were acquired gradually. On the other hand, some investigators (the present experiment; Glazer \& Weiss, 1976b; Maier \& Testa, 1975) found that the inescapably shocked rats frequently escaped as well as the controls on the first few trials and escaped increasingly more slowly as the session progressed. Moreover, these investigators frequently found that the interference effect would occur even in paradigms in which there was no tendency for the controls to improve across trials. Although the shapes of these acquisition curves are not inconsistent with the learned helplessness hypothesis, they are not clearly predicted from the hypothesis as formulated. Thus, the learned helplessness hypothesis will require modification to more adequately incorporate these findings.

In supporting the learned helplessness hypothesis, I do not intend to imply that all interference effects caused by exposure to inescapable shocks in rats are examples of learned helplessness. Competing responses and alterations in brain neurochemistry can interfere with escape from shock (e.g., Bracewell \& Black, 1974; Maier, 1970; Weiss et al., 1975). I would argue, not that these variables are unimportant, but that it is also possible to produce an interference effect which is less dependent on these variables than it is on having learned that active responses are ineffectual in controlling shock.

\section{REFERENCES}

Anisman, H. Time-dependent variations in aversively motivated behaviors: Nonassociative effects of cholinergic and catecholaminergic activity. Psychological Review, 1975, 82, 359-385,

Anisman. H., \& Waller, T. G. Effects of inescapable shock on subsequent avoidance performance: Role of response repertoire changes. Behavioral Biology, 1973, 9, 331-355.

Bliss, E. L., Allion, J., \& ZWanziger, J. Metabolism of norepinephrine, serotonin, and dopamine in rat brain with stress. 
Journal of Pharmacology and Experimental Therapeutics, 1968, 164. 122-134.

Bracewell, R. J., \& Black, A. H. The effects of restraint and noncontingent preshock on subsequent escape learning in the rat. Learning and Motivation, 1974, 5, 53-69.

GLAZER, H. I., \& WeIss, J. M. Long-term and transitory interference effects. Journal of Experimental Psychology: Animal Behavior Processes, 1976, 2, 191-201. (a)

Glazer, H. I., \& WEIsS, J. M. Long-term interference effect: An alternative to "learned helplessness." Journal of Experimental Psychology: Animal Behavior Processes, 1976, 2, 202-213. (b)

Hays, W. L., \& WinkLeR, R. L. Statistics: Probability, inference and decision. New York: Holt, Rinehart, \& Winston, 1971.

LEVIS, D. J. Learned helplessness: A reply and an alternative S-R interpretation. Journal of Experimental Psychology: General, 1976, 105, 47-65.

MAIER, S. F. Failure to escape traumatic electric shock: Incompatible skeletal-motor responses or learned helplessness? Learning and Motivation, 1970, 1, 157-169.

Maier, S. F., \& Seligman, M. E. P. Learned helplessness: Theory and evidence. Journal of Experimental Psychology: General, 1976, 105, 3-46.

Maier, S. F., \& Testa, T. J. Failure to learn to escape by rats previously exposed to inescapable shock is partly produced by associative interference. Journal of Comparative and Physiological Psychology, 1975, 88, 554-564.

Maier, S. F., Albin, R. W., \& Testa, T. J. Failure to learn to escape in rats previously exposed to inescapable shock depends on nature of escape response. Journal of Comparative and Physiological Psychology, 1973, 85, 581-592.

MCClelland, W. J., \& Colman, F. D. Activity and different types of electric shock stimuli. Psychonomic Science, 1967, 7, 391-392.

Seligman, M. E. P., \& Beagley, G. Learned helplessness in the rat. Journal of Comparative and Physiological Psychology, 1975, 88, 534-541.

Seligman, M. E. P., \& Maier, S. F. Failure to escape traumatic shock. Journal of Experimental Psychology, 1967, 74, 1-9.

Seligman, M. E. P., Rossellini, R. A., \& Kozak, M. J. Learned helplessness in the rat: Time course, immunization, and reversibility. Journal of Comparative and Physiological Psychology, 1975. 88. 542-547.

Stone. E. A. Adrenergic activity in rat hypothalamus following extreme muscular exertion. American Journal of Physiology, 1973, 224, 165-169.

WEISS, J. M. Effects of coping behavior in different warning signal conditions on stress pathology in rats. Journal of Comparative and Physiological Psychology, 1971, 77, 1-13.

Weiss, J. M., Glazer, H. I., \& Pohorecky, L. A. Coping behavior and neurochemical changes: An alternative explanation for the original "learned helplessness" experiments. In Relevance of the psychopathological animal model to the human. New York: Plenum Press. 1975.

(Received for publication July 22, 1976; revision accepted December 1, 1976.) 